http://jmscr.igmpublication.org/home/ ISSN (e)-2347-176x ISSN (p) 2455-0450 crossref DOI: https://dx.doi.org/10.18535/jmscr/v7i7.109

\title{
Basic Physics \& Principles of Imaging by Computed Tomography
}

\author{
Author \\ Dr Shah Md. Ejajul Islam ${ }^{1^{*}}$ \\ ${ }^{1}$ MBBS. DNM (DU), Assistant Professor, Nuclear Medicine, Dhaka Medical College, Bangladesh \\ *Corresponding Author \\ Dr Shah Md. Ejajul Islam
}

\begin{abstract}
Computed Tomography $(C T)$ is a radiological imaging technique for producing cross-sectional tomographic images. At present it is one of the most useful imaging modality in diagnostic medicine. The main goal of this study to identify the basic physics \& principles of imaging by computed tomography. This study was a descriptive study. Data were collected from different books, journal articles and internet sources. CT provides tomographic section where different body structure is not superimposed like conventional radiography. Image quality contrast and resolution of CT image are very high. Size, location and extension of pathological lesion can be determined with extreme accuracy. Density difference as low as $0.5 \%$ can be easily distinguish by $C T$. CT is one of the most useful modality in diagnostic medicine. Although there are some limitations, CT becomes more and more acceptable day to day in our country due to better diagnostic value.

Keywords: CT, Cross-sectional tomographic images, Pathological lesion.
\end{abstract}

\section{Introduction}

Modern Medical science has great attraction with imaging for diagnosis of diseases. These images constitute the concise illustration of information of diseases. CT is a radiological imaging technique for producing cross-sectional tomographic images. Following invention of x-ray, CT was the most advance invention in 1972. Now a day it is one of the most useful imaging modality in diagnostic medicine. Though CT is well known to all, but it should be noted that like conventional radiograph, CT image is not formed directly by transmitted $\mathrm{x}$ ray beam. So the purpose of this article is to provide knowledge \& information about this wonderful imaging modality among all the fields of medical science. The main goal of this study to identify the basic physics \& principles of imaging by computed tomography.

\section{Basic principles of CT}

The basic principles of computed tomography are differ from conventional radiography and may be stated as below-

a) Scanning of a slice of tissue by multiple angles with $\mathrm{x}$-ray beam. Usually wide fan shaped beam is used now instead previously used narrow beam.

b) Transmitted beam, which constitute the concise illustration of information was counted by a suitable detectors (usually scintillation detectors).

c) Fed on a computer, which calculate a 
relative linear attenuation coefficient $(\mu)$ in each section various tissue element (voxel) for each pixel.

d) Calculating a relative linear attenuation coefficient $(\mu)$, the number is normalized to a reference material (water), magnified to a larger whole number (integer) and reported as a new number, called CT number that recorded in the pixel of picture matrix.

e) Finally image is displayed as gray scale image after a mathematical reconstruction by analytical method which is proportional to CT number.

\section{Historical Background}

In April of 1972, G. N. Hounsfields, a senior research scientist at EMI limited in Middlesex, England was first announced the invention of a revolutionary new imaging technique, which is called computerized axial transverse imaging. To shortening the scanning time \& to improve the image quality CT is undergone multiple steps of development.

First generation- The original EMI scanner was designed by a pencil like beam of single $\mathrm{X}$ ray tube above $\&$ a single detector, that set on a gantry with their proper alignment. The gantry was processed both linear and rotatory motion and scanned was done on different angles. The total number of transmission measurement was the product of the number of linear measurements (160) \& the number of rotator steps (180), which were 28800 in original EMI scanner. The scan time for each tomographic section was 4.5-5 minutes. So total scan times for multiple sections required for a complete clinical study was about 25-30 minutes.

Second generation (1977)- lt was designed by single fan shaped $\mathrm{x}$-ray beam (instead of pencil like beam) and multiple number of detectors (about 30). Both $\mathrm{x}$ - ray tube \& detectors were aligned properly. They were processed both linear and rotatory motion and scanned was done on different angles. So second generation scanner produces a tomography section in between 10-90 sec depend on manufacturer.
Third generation (1983)- It was designed by single much wider fan shaped $\mathrm{X}$ ray beam (than a second generation unit), that wide enough to encompass the entire width of patient and more number of detectors (about 300). Both X ray tube $\&$ detectors were aligned properly in a gantry, which had no linear movement, but $360^{\circ}$ rotatory movement. Each rotation completes one tomography section. So scan times for third generation scanners ranges from 2-10 seconds.

Fourth generation (1983) - It was designed by a ring of detectors completely surround the patient. Only $\mathrm{X}$ ray tube rotates through a $360^{\circ}$ arc with a wide fan beam and the detectors remain stationary. As the fan beam revolves, it activates the detector that fall in its path and each rotation of tube completes one tomography section. Fourth generation scanner are not faster in principle than third generation, but their major advantage is easier detector calibration.

Fifth generation (announced in spring 1983) - It was designed by a fixed ring of detectors completely surround the patient. Multiple X ray tube of different position also fixed that would be fired sequentially at a ring of fixed detectors, and tomographic section would be completed in a few milliseconds.

The basic components of $\mathrm{CT} X$ ray TubesConventional $\mathrm{X}$ ray tubes are currently being used in all commercial scanners. Ideally the radiation source for CT would supply a monochromatic Xray beam. In a monochromic beam image construction is simpler and more accurate. As Xray is a polychromic in nature, by proper collimation \& filtration almost monochromic beam is prepared for image construction. Earlier models used oil cooled, fixed anode, relatively large focal spot ( $2 \mathrm{~mm} \mathrm{X} \mathrm{16mm),} \mathrm{at} \mathrm{energy} \mathrm{of} 120$ kilovolt and $30 \mathrm{~mA}$. Recently Special type of $\mathrm{X}$ ray tube have been developed for CT. Newer models are air cooled, rotating anode, cylindrical target, small focal spot $(0.6 \mathrm{~mm})$, much higher current $600 \mathrm{~mA}$. In $3^{\text {rd }} \& 4^{\mathrm{m}}$ generation CT scanner more than 300 separate radiation pulse may be delivered during the circular motion to produce one tomographic 
section, The tube moves continuously, even though the radiation is pulsed. The computer is programmed to compensate for the motion. Each pulse lasts 2-3 msec, so actual time of radiation for each scan is less than one second ( $3 \mathrm{msec} /$ pulse $\mathrm{X}$ 300 pulses $=0.9 \mathrm{sec}$ ), which is about $20 \%$ of total time $(5 \mathrm{sec})$. The advantages of pulse system are the following-

1. Much larger quantities of radiation can be delivered in a short time, so scan time is less.

2. Smaller focal spots allow much more accurate collimation which results in lower patient exposure.

3. Electronic circuitry can be recalibrated between pulses; so x-ray output is more accurately controlled.

4. The pulse length can be accurately regulated to optimized counting statistics and detector response.

\section{Collimators}

Collimation of $\mathrm{X}$ ray beam occurs at two points: (a) close to the $X$ ray tube $\&$ (b) at the detector with proper alignment between them. Collimators control the scattered radiation; regulate the thickness of voxel (3-13 $\mathrm{mm})$ and with proper filter made up photon almost monochromic nature. The pixel size is determined by the computer program.

\section{Detectors}

Transmitted beams are counted by suitable detectors. The most important characteristics of CT detectors are low cost, high efficiency, high stability and rapid responsiveness. Two types of detectors are currently used in CT scanner- (a) scintillation crystal detectors, which have a high counting efficiency but suffer from nonlinear response and afterglow \& (b) Gas filled detector, made by pressurized xenon, which have linear response and do not suffer from afterglow but are less efficient.

Usually scintillation crystal detectors are used in CT scanners. The principal advantage sodium iodide crystals are almost $100 \%$ efficient in the diagnostic energy range. But important disadvantage is afterglow, or phosphorescence after the signal has passed, produces a serious problem. For instance, bismuth-germinate combines the desirable feature of high efficiency and no afterglow.

\section{Computer}

Computer plays important role for rapid data analysis Image reconstruction. Computer is related to following component-

1) Input area- Where $x$-ray tubes and detectors are set on a gantry with proper alignment. The patient is also properly set within this alignment.

2) Operating console- It is the command area of computer, where the computer can previously programmed for proper tomography.

3) Electronic circuits- Which are used for energy discrimination by analysis of pulse height and supply only informative data to the CPU.

4) CPU- which is used for collection, analysis \& storage of informative data and supply output signal for formation of visible image

5) Display device- Where reconstructed image is displayed as gray scale image.

Steps of image reconstruction- CT images are reconstructed by complex mathematical process. In EMI scanner mathematical reconstruction were done with iterative method, but now a day reconstruction of images are usually done by analytic method by using either two dimensional Fourier analysis, filtered back projection or combination of these two processes. Analytic methods start computation as soon as the first ray projection is scanned, so they are fast and the reconstruction is completed within a few second after all the data are collected by following steps.

\section{Calculation of Linear attenuation coefficient} $(\boldsymbol{\mu})-$, In computed tomography a cross-sectional layer of body is divided in to many tiny blocks and then each blocks are assign a number 
proportional to the degree of attenuation of x-ray beam. The individual blocks are called voxel. Linear attenuation coefficient $(\mu)$ is used to quantitate the attenuation, which depend on the density \& the thickness of voxel and the quality of the beam. The individual block of tissue (voxel) is represented in to unit square of image matrix of computer which is called pixel. The original EMI scanner unit took 28800 readings (160 linear readings $\mathrm{X} 180^{\circ}$ ) and fan beam scanner may take as many as 90000 readings (300 pulse X 300 detectors) for each tomographic section,

Linear attenuation coefficient is calculated by an

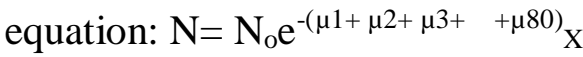

Where, No $=$ Number of initial photon, $\mathrm{N}=$ Number of transmitted photon, $X=$ thickness $\& \mu$ $=$ linear attenuation coefficient.

Correction factors: Linear attenuation coefficient $(\mu)$ should be precisely corrected, both in absolute and relative value from pixel to pixel. Two correction factors are incorporated in to CT program.

a) Prevention of alteration in linear attenuation coefficient- Linear attenuation coefficient is constant in monochromic beam but due to polychromic nature of beam it is changed in same element produced an error, that can be corrected by specific computer programmed, that back to it constant level.

b) Weighting factor- Weighting factor is a correction program that used to compensate for the difference in size in between the actual element and that seen by perpendicular and oblique beams which recorded in pixel of picture matrix.

2. Calculation of CT number- After calculation of Linear attenuation coefficient $(\mu)$ of each pixel, the data is normalized to a reference material (Water), magnified to a larger whole number (integer) and reported as a new number, called CT number, that recorded in the pixel. Dense bone is assigned the highest value $(+1)$, air the lowest value (-1) and water a value of 0 . In original EMI scanner, magnifying constant was considered as 500 , therefore dense bone was +500 , water 0 , and air -500. Nevertheless, newer units have more expanded scale employing a magnifying constant of 2000 or more. Mathematically CT number can be calculated by following equation. $\mathrm{CT}$ number $=$ $\mathrm{K}\left(\mu_{\mathrm{p}}-\mu_{\mathrm{w}}\right) / \mathrm{n}_{\mathrm{w}}$ where, $\mathrm{K}=$ magnifying constant $\left(\mu_{\mathrm{p}}=\right.$ linear attenuation coefficient of pixel $\& \mu_{\mathrm{w}}=$ Linear attenuation coefficient of water.

\section{Display unit}

The mathematical reconstruction is displayed either on a paper as a number, called CT number which are proportional to linear attenuation coefficient of the tissue in the tomographic section or as a gray scale image in a CRT which are proportional to the CT number. Contrast levels can be adjusted to augmented specific areas in the image. The difference in the linear attenuation coefficient of soft tissues, excluding fat, is about $4 \%$, with fresh blood clot among the densest and cerebrospinal fluid the least dense. This 4\% difference can easily be demonstrated when these structures are sufficiently large and appropriately located.

\section{Image quality}

Image quality (clarity) may be defined as the visibility of diagnostically important structure in the CT images. Usually following interrelated characteristics that determined the quality of CT image.

Precision- Precision in computed tomography is a measure of background or matrix uniformity. If a perfectly homogenous object such as water bath is examined with a CT scanner, all element in the picture matrix should have exactly the same CT number. Deviations from uniformity represent statistical fluctuation, called quantum mottle. A lack of precision or presence of mottle is the limiting factor of CT performance.

Contrast- Contrast is the most important feature of an image. Contrast may be defined as the percentage difference between the object of interest and the background area. The amount of 
contrast required for visibility depend on number of factors: object size, matrix size, field size, pixel size \& image noise, Resolution- Resolution of an image may be defined as the ability of accurate representation of an object. This may be affected by blur. So the term resolution is often used to describe the blur characteristics of an imaging system. Blur may be produced by motion of patient, collimators blur \& Intrinsic blur of non linear response of scintillation detector. In CT, with high subject contrast and good precision, resolution is approximately one and half times of pixel size. The more homogenous the background, better resolution of low contrast image.

Spatial uniformity- Spatial uniformity refers to the accuracy of reading over an area of the matrix relative to another area. Breaks in spatial uniformity may be distorted the size, shape and location of image.

Image noise- The most serious factor limiting image quality is statistical noise. This problem can partially solved by increasing scan time (more radiation) and picture element (pixel size), but both of these alternatives have produced adverse effect. Increasing scan times increases patient exposure and increasing pixel size decrease high contrast resolution.

\section{Proper technique and command- Well} preparation, proper positioning, immobilization and uniform exposure of patient affect the image quality. Selection of slice thickness, pixel size, proper use of contrast media, proper calibration of detectors and mechanical performance increase quality of images.

\section{Artifacts}

1) Motion artifact- Motion has a devastating effect on image quality in CT. The resulting scan contains Black and white vertical bands are noted in side to side motion, and diagonal band for rotatory motion.

2) High density foreign material- It will produced star pattern of artifact.

3) Detectors artifact- Ring pattern of artifacts are produced by faulty detector calibration.

4) Volume averaging artifact- Calculated linear attenuation coefficient is a weighted average of all material in the voxel. So even a small amount of calcium in a voxel represented the whole pixel and appears bony density in the image.

5) Geometric oversights artifact- In CT two scan slice are partially overlapped and recorded the same area twice.

\section{Discussion}

\section{Usefulness \& limitation}

Usefulness- CT provides tomographic section where different body structure is not superimposed like conventional radiography. Image quality contrast and resolution of CT image are very high. Size, location and extension of pathological lesion can be determined with extreme accuracy. Density difference as low as $0.5 \%$ can be easily distinguish by CT. Image is reconstructed by computer, so it can be store, displayed, compared and manipulated in many way for diagnosis, follow-up \& research purposes.

\section{Limitations}

Although CT has many advantages, it also has some limitations. Machinery cost is very high, so it is not easily available. Examination cost is also very high, so it is unbearable for maximum population. It provides only anatomical image and cannot provide functional image. Real rime imaging is not possible by CT. Computed tomography can not reveal any pathological lesion less than $1 \mathrm{~mm}$.

\section{Conclusion}

Currently CT is one of the most useful modality in diagnostic medicine. Although there are some limitations, CT becomes more and more acceptable day to day in our country due to better diagnostic value. Its benefit should be reached to all people irrespective of socio-economic 
condition in near future for better service of humanity.

\section{References}

1. Christensen's Introduction to the physics of diagnostic radiology by Tomas S. Curry 111, James E. Dowdey, Robert C. Murry 3rd Edition Page: 320-350

2. EMI Scanner: A New Perspective on Brain Tissue. Middlesex, England, EMI Limited, 1973.

3. Hounsfield, G. N.: Picture quality of computed tomoyaphy Am. J. Roentgenol. 127:3,1976.

4. McCullough, E.C. and Payne J.T. : X ray transmission computed tomoyaphy. Med. Phys.-4:85 1977.

5. Brooks, R. A. and DiChiro G.: Theory of image reconstruction in computed tomography. 117:561,1975. 